\title{
Internal kinematics of isolated modelled disc galaxies
}

\author{
W. Kapferer ${ }^{1}$, T. Kronberger ${ }^{1,2}$, S. Schindler ${ }^{1}$, A. Böhm² ${ }^{2}$ and B. L. Ziegler ${ }^{2}$ \\ 1 Institut für Astrophysik, Leopold-Franzens-Universität Innsbruck, Technikerstr. 25, 6020 Innsbruck, Austria \\ e-mail: wolfgang.e.kapferer@uibk.ac.at \\ 2 Institut für Astrophysik, Universität Göttingen, Friedrich-Hund-Platz 1, 37077 Göttingen, Germany
}

Received 5 August 2005 / Accepted 12 September 2005

\section{ABSTRACT}

\begin{abstract}
We present a systematic investigation of rotation curves (RCs) of fully hydrodynamically simulated galaxies, including cooling, star formation with associated feedback, and galactic winds. Applying two commonly used fitting formulae to characterize the RCs, we investigated systematic effects on the shape of RCs by both the observational constraints and internal properties of the galaxies. We mainly focused on effects that occur in measurements of intermediate and high redshift galaxies. We found that RC parameters are affected by the observational setup, like slit misalignment or the spatial resolution and that they also depend on the evolution of a galaxy. Therefore, a direct comparison of quantities derived from measured RCs with predictions of semi-analytic models is difficult. The virial velocity $V_{\mathrm{c}}$, which is usually calculated and used by semi-analytic models, can differ significantly from fit parameters like $V_{\max }$ or $V_{\mathrm{opt}}$ inferred from RCs. We found that $V_{\mathrm{c}}$ is usually lower than typical characteristic velocities derived from RCs. $V_{\max }$ alone is in general not a robust estimator of the virial mass.
\end{abstract}

Key words. galaxies: kinematics and dynamics - galaxies: spiral - galaxies: structure

\section{Introduction}

Spatially resolved rotation curves (RCs) are a fundamental tool for studying the internal kinematics and distribution of mass in spiral galaxies. The first discrepancy between the differential Kepler-type of rotation and the rotation of galaxies was detected by Babcock (1939). These first indirect measurements of non-luminous matter introduced the concept of dark matter (DM) into astrophysics. An overview is given by Sofue \& Rubin (2001, and references therein).

An important application of RCs lies within a correlation of the luminosity and the maximum rotational velocity of spirals found by Tully \& Fisher (1977). The physical origin of the slope and the scatter of the TFR are subjects still being debated. Different theoretical approaches exist that differ mainly in their predictions of the redshift evolution of the TFR. Therefore, Ziegler et al. (2002) and Böhm et al. (2004) used a sample of field galaxies in the FORS Deep Field to study the TFR at intermediate redshift. They find a significant change of slope in comparison to local samples, which is mainly caused by small, star-forming distant galaxies. However, the measurement of rotational velocities is more complicated in the case of distant, apparently small spirals. In this work we investigate how parameters from models that describe the shape of RCs are influenced by observational constraints and by internal properties of galaxies. Only techniques that take these systematics into account, as e.g. the method presented in Böhm et al. (2004), can get robust results for $V_{\max }$.
In recent years, fully $N$-body/hydrodynamic simulations of spiral galaxies have become an important tool for understanding the formation and evolution of spiral galaxies (e.g. Mihos \& Hernquist 1994; Springel \& Hernquist 2002). In these simulations, cooling, stellar feedback, and galactic winds are taken into account to model galaxies in a physically motivated way. Here, we extracted RCs from model galaxies simulated with GADGET2 (Springel 2005). We investigated the influence of large relative slit widths, inclinations, and slit misalignments on the determination of fitting parameters like $V_{\text {opt }}$ or $V_{\max }$ for intermediate and high redshift galaxies. These parameters are commonly used (e.g. Courteau 1997; Yegerova \& Salucci 2004) as a measure of the "peak" circular velocity, e.g. when determining Tully-Fisher relations.

\section{Simulations}

Recently Kapferer et al. (2005) studied the influence of galaxyinteractions on the strength and evolution of the star-formation rate of the interacting system. In the present work, we investigate the isolated model galaxies presented in Kapferer et al. (2005). The initial conditions (hereafter ICs) of the model galaxies were built according to Springel et al. (2004), which is based on the work of Mo et al. (1998). The two model galaxies in this work were chosen such that they represent a Milky Way type and a small spiral galaxy without any bulge component. The properties of the ICs of the model galaxies are listed in Table 1 . The combined $N$-body/SPH simulation then calculates 
Table 1. Properties of the initial conditions of the model galaxies.

\begin{tabular}{|c|c|c|}
\hline Properties & Galaxy A & Galaxy B \\
\hline Circular velocity $V_{\mathrm{c}}{ }^{1}$ & 160 & 80 \\
\hline Disc mass fraction ${ }^{2}$ & 0.05 & 0.05 \\
\hline Gas content in the disc ${ }^{3}$ & 0.25 & 0.25 \\
\hline Disc thickness ${ }^{4}$ & 0.02 & 0.02 \\
\hline Total mass $\left[M_{\odot}\right]$ & $1.33 \times 10^{12} h^{-1}$ & $1.67 \times 10^{11} h^{-1}$ \\
\hline Disc scale length [kpc] & $4.51 h^{-1}$ & $2.25 h^{-1}$ \\
\hline
\end{tabular}

1 Circular velocity at $r_{200}$ in $\mathrm{km} \mathrm{s}^{-1} .{ }^{2}$ Fraction of disc particles (stars/gas) in units of halo mass. ${ }^{3}$ Relative content of gas in the disc.

${ }^{4}$ Thickness of the disc in units of radial scale length.

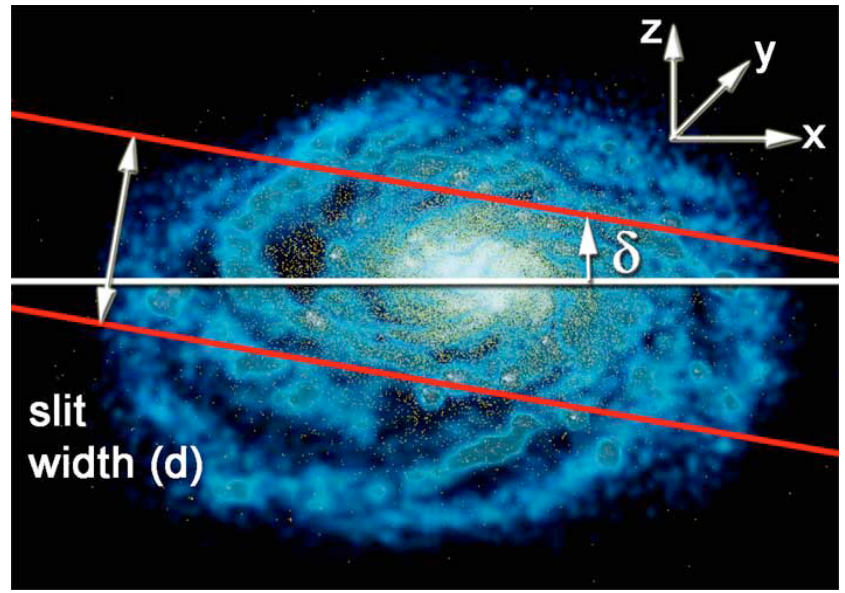

Fig. 1. Image of model galaxy A and a virtual slit for extracting a rotation curve. The slit width $d$ and the slit misalignment angle $\delta$ are indicated.

5 Gyr of isolated evolution. For every time step, we know the velocity of each particle and can hence extract realistic rotation curves.

\subsection{Rotation curve extraction}

In order to extract the rotation curves (RCs) of our simulated model galaxies, we defined a slit with a width $d$, see Fig. 1. In addition we allowed for a misalignment angle $\delta$ to simulate rotations of the slit with respect to the major axis of the system. Such rotations sometimes occur in observations using multiobject spectroscopy. In Fig. 1 the different parameters for the slit are shown. The slit width $d$ and the misalignment $\delta$ of the slit with respect to the major axis define the virtual slit. We extracted the RCs from the velocity field of the gas in the following way. The velocity as a function of radius was determined by averaging over all line-of-sight velocities of gas particles in thin bins, with a bin size $\Delta r$ along the slit and a side length $d$ perpendicular to the spatial axis. The velocity field taken for the RC extraction is shown in Fig. 2a. In Fig. 2b the spatial sampling along the slit is sketched, while an $\mathrm{RC}$ with a resolution of $0.1 \mathrm{kpc}$ was extracted and used as a reference.

In order to determine systematic effects of large slit widths $d$ (relative to the galaxy size), which occur in the case of observations of distant $(z \approx 0.5)$ galaxies (e.g. Böhm et al. 2004) we varied $\mathrm{d}$ in a range of several kpc. Not only does the relative slit
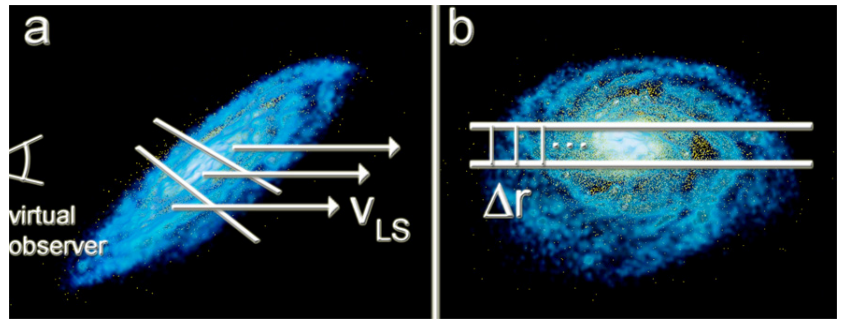

Fig. 2. Sketch of our procedure to extract rotation curves of the model galaxies. a) the line-of-sight velocity field of a model galaxy is indicated as it would be observed by a virtual observer. b) the sampling along the slit is highlighted $(\Delta r)$.

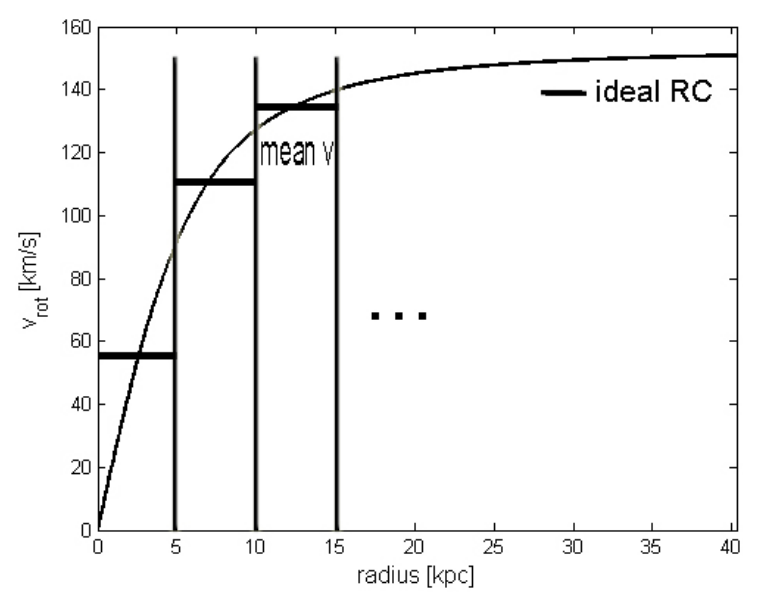

Fig. 3. An example for a $5 \mathrm{kpc}$ binning of the ideal RC.

width vary in observations of distant disc galaxies, but so does the sampling of the velocity field along the spatial axis (i.e. the spatial resolution). In order to simulate this finite spatial resolution, we binned the reference RC with different bin sizes. In Fig. 3 we show the extraction of different RCs, corresponding to different spatial resolutions along the slit.

\section{Results}

\subsection{Rotation curves as a function of evolution}

As a first step, we investigated the RCs of the ICs and compared them to the RCs of the fully hydrodynamically treated galaxies after 5 Gyr of evolution. Note that the ICs are based on an analytic model introduced by Mo et al. (1998). The evolution starting from these ICs is determined by the influence of the dynamics, the star formation with feedback, and stellar winds of the system. Therefore the RCs and other internal properties of the evolved galaxies do differ from those of the ICs. The general evolutionary trend of the RCs is presented in Fig. 4. The rotation curves were obtained by setting a slit width of $4 \mathrm{kpc}$ (galaxy A) and $1 \mathrm{kpc}$ (galaxy B) without any slit misalignment $\delta$. The galaxies were inclined with an inclination angle of $i=80^{\circ}$. A spatial resolution of $0.1 \mathrm{kpc}$ was adopted to extract ideal RCs. It is clearly visible that the rotational velocities get lower for the evolved galaxies. The decrease in the overall angular momentum of gas particles in the disc can be explained by mass ejection due to galactic winds and the rearrangement of the gas in the disc due to the fully hydrodynamic 


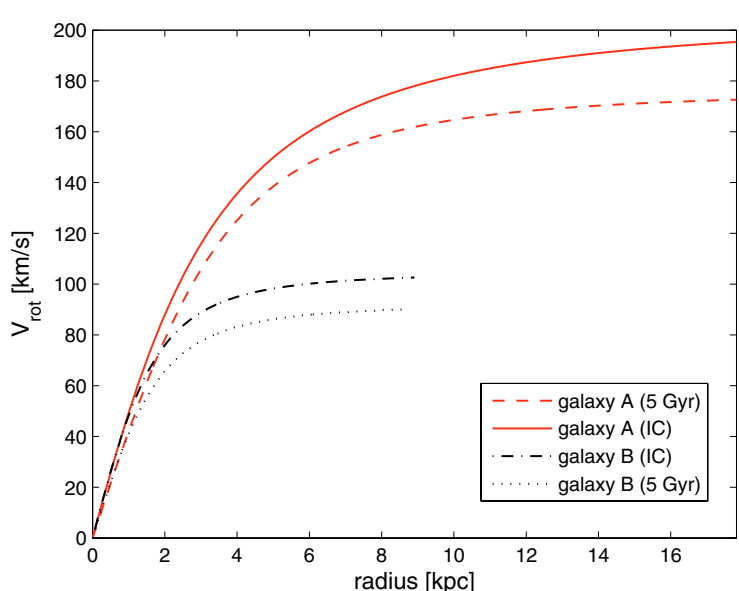

Fig. 4. Best fits to the measured RCs of model galaxies A and B. The fits are done for the ICs and for the evolved (5 Gyr) systems.

treatment as the disc rotates. As we wanted to investigate the dependencies on different observational constraints, e.g. galaxy alignment with respect to the spectroscopic slit or slit misalignment, we used models hereafter to describe the shape of the RC. Physically motivated fitting functions are used in observational work, such as the universal rotation curve (URC) Eq. (2) (Persic et al. 1996) or purely phenomenological fitting formulae like Eq. (1) (Courteau 1997). Although we are aware that Eq. (1) cannot reproduce the many observed declining RCs, it is suitable for our model galaxies, which do not decline in the outer parts.

The RCs shown in Fig. 4 are best fits to the measured rotational velocities of the model galaxies using the fitting formula of Courteau (1997). This function is defined as

$V_{\mathrm{rot}}(r)=\frac{V_{\max } r}{\left(r^{a}+r_{0}^{a}\right)^{\frac{1}{a}}}\left[\mathrm{~km} \mathrm{~s}^{-1}\right]$,

where $r$ is the galactocentric distance, and $r_{0}$ and $a$ are free fitting parameters. Clearly, with the general decrease in the rotational velocities $V_{\max }$ also gets lower. The physically motivated URC (Persic et al. 1996) is a superposition of the velocity field of the DM halo and the disc. The URC can be expressed as follows

$$
\begin{aligned}
V_{\mathrm{URC}}\left(\frac{r}{r_{\mathrm{opt}}}\right)= & V\left(r_{\mathrm{opt}}\right)\left\{\left(0.72+0.44 \log \left(\frac{L}{L_{*}}\right)\right)\right. \\
& \times \frac{1.97 x^{1.22}}{\left(x^{2}+0.78^{2}\right)^{1.43}}+1.6 \exp \left[-0.4 \frac{L}{L_{*}}\right] \\
& \left.\times \frac{x^{2}}{x^{2}+1.5^{2}\left(\frac{L}{L_{*}}\right)^{0.4}}\right\}^{\frac{1}{2}}\left[\mathrm{~km} \mathrm{~s}^{-1}\right],
\end{aligned}
$$

where $x=r / r_{\mathrm{opt}}, V_{\mathrm{opt}}$ is the rotational velocity at $r_{\mathrm{opt}}$ (the reference scale, which for an exponential disc is $3.2 R_{\mathrm{d}}$ ), and $L$ the absolute blue luminosity of the galaxy. In Fig. 5 the URC fit to our data is shown together with the Courteau (1997) fit. Both functions represent the RCs of the model galaxies very well. Although we are aware that the Courteau fitting function has no physical justification, we use both functions to fit our data,

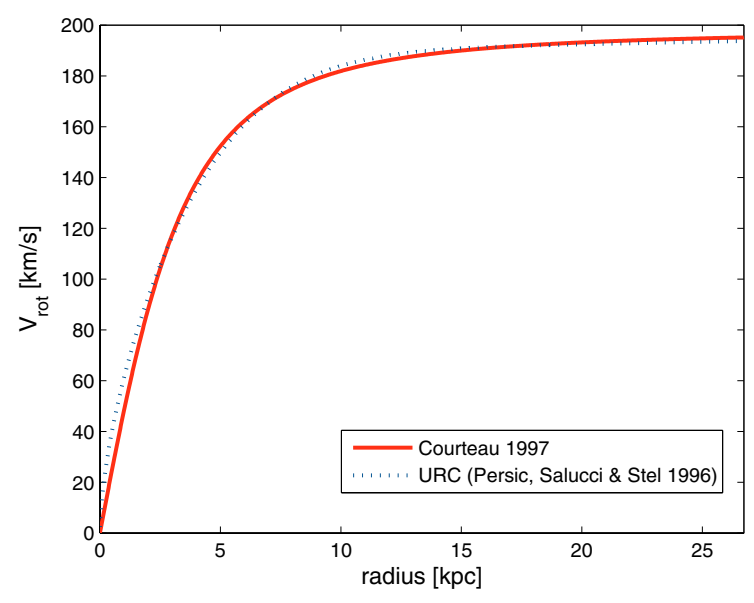

Fig. 5. Comparison of the two profiles (Courteau, URC) for model galaxy A. Both profiles represent the velocity field very well.

Table 2. Fitting parameters for Eq. (1) (Courteau 1997) for RCs of the ICs and the fully hydrodynamically treated galaxies (evolution 5 Gyr).

\begin{tabular}{cccc}
\hline \hline Fitting & Parameter & Galaxy A & Galaxy B \\
\hline I & $V_{\max }\left[\mathrm{km} \mathrm{s}^{-1}\right]$ & $205_{-3}^{+3}$ & $105_{-0.8}^{+1.8}$ \\
I & $r_{0}[\mathrm{kpc}]$ & $3.91_{-0.14}^{+0.14}$ & $1.83_{-0.08}^{+0.07}$ \\
I & $a$ & $1.62_{-0.11}^{+0.11}$ & $1.89_{-0.15}^{+0.51}$ \\
\hline II & $V_{\max }\left[\mathrm{km} \mathrm{s}^{-1}\right]$ & $169.67_{-4.57}^{+4.53}$ & $91.8_{-2.36}^{+2.2}$ \\
II & $r_{0}[\mathrm{kpc}]$ & $4.6_{-0.47}^{+0.4}$ & $2.05_{-0.17}^{+0.17}$ \\
II & $a$ & $3.39_{-1.23}^{+1.24}$ & $2.14_{-0.4}^{+0.41}$ \\
\hline
\end{tabular}

I: Initial conditions.

II: Evolved galaxies ( $t=5 \mathrm{Gyr})$.

Table 3. Parameter $V_{\text {opt }}$ for Eq. (2) (Persic et al. 1996) for RCs of the ICs and the fully hydrodynamically treated galaxies (evolution $5 \mathrm{Gyr}$ ).

\begin{tabular}{cccc}
\hline \hline Fitting & Parameter & Galaxy A & Galaxy B \\
\hline I & $V_{\text {opt }}\left[\mathrm{km} \mathrm{s}^{-1}\right]$ & $186.5_{-0.8}^{+0.8}$ & $80.3_{-2.3}^{+2.3}$ \\
I & $L / L_{*}$ & 0.61 & 0.075 \\
\hline II & $V_{\text {opt }}\left[\mathrm{km} \mathrm{s}^{-1}\right]$ & $183.2_{-6}^{+6}$ & $91.1_{-2.2}^{+2.2}$ \\
II & $L / L_{*}$ & 0.65 & 0.08 \\
\hline
\end{tabular}

I: Initial. conditions.

II: Evolved galaxies ( $t=5 \mathrm{Gyr})$.

in order to investigate the shape of the RC as a function of the observational setup, i.e. galaxy alignment and slit properties.

The fit parameters for Eqs. (1) and (2) with 95\% confidence level errors are listed in Tables 2 and 3, respectively. Note that no restrictions were set on the fit parameters ( $V_{\max }$, $r_{0}$ and a for the Courteau function and $V_{\text {opt }}$ for the URC). The blue band luminosity $L_{B}$ was estimated from the stellar mass assuming a stellar mass to light ratio of 1.2 (mean from different star-formation histories in the redshift range $z=0.5-1.4$, Dickinson et al. 2003). The $L_{*}$ luminosity at $z=0.5$ was adopted as $M_{B}^{*}=-21.3$ (Gabasch et al. 2004), which corresponds to $\log \left(L_{B}^{*}\right)=10.72$. We find values for $L / L_{*}$ in the range of 0.61-0.65 for model galaxy A and 0.075-0.08 for model galaxy B. An evolution of $L / L_{*}$ that is due to new stars forming in the galaxies can be seen. For the investigations of the $\mathrm{RC}$ as a function of observational bias, we choose $L / L_{*}$ as an 


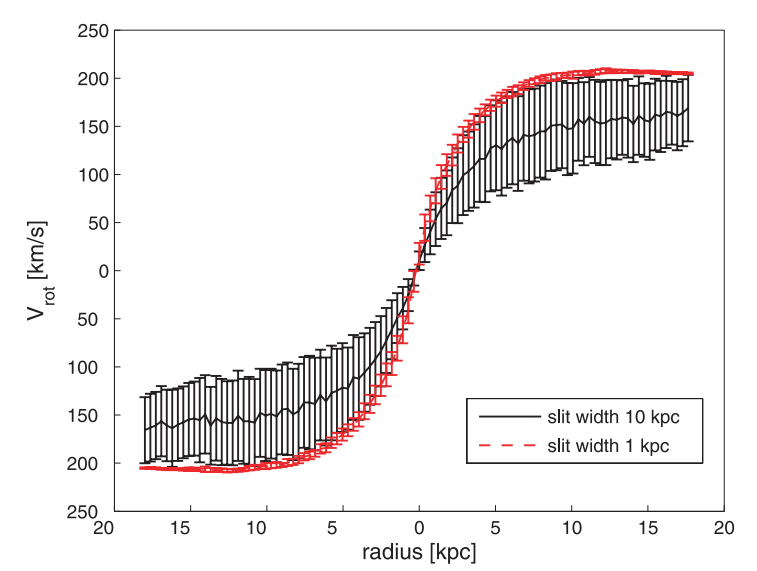

Fig. 6. RCs of model galaxy A ICs, for two different slit widths (1 kpc and $10 \mathrm{kpc}$ ). The error bars indicated in the figure are the standard deviations of the mean velocity in each $0.1 \mathrm{kpc}$ bin, see Fig. 2 (image b).

additional free parameter, to get better representations of the RCs.

Most present models of galaxy formation and evolution rely on the work of Mo et al. (1998). Indeed, this model can correctly reproduce the general shape of rotation curves, but galaxy evolution can alter the RC. Note especially the differences between $V_{\mathrm{c}}$ and $V_{\mathrm{max} / \mathrm{opt}}$ from Tables 1-3, respectively. A comparison of these semi-analytic models and observations is generally complicated by the fact that $V_{\max / o p t}$ is determined differently from $V_{\mathrm{c}}$. In the case of mass reconstruction via RCs, the superposition of the velocity field of the halo and the disc in the URC ansatz would be the adequate approach.

\subsection{Rotation curves as a function of the slit width for model galaxy $A$}

In order to study systematic effects of large relative slit widths as they appear in observations of galaxies at intermediate and high redshift, we extracted the RC of model galaxy A for several slit widths. Of course, slit widths for local spiral systems are orders of magnitudes smaller, but for observations of galaxies in the redshift range 0.5 to 1 , as carried out in a project related to the present work (Ziegler et al. 2003), typical slit widths become comparable to the disc scale length $\mathrm{R}_{\mathrm{d}}$. It is important to note that such large slit widths result in an integration of the velocity field perpendicular to the spatial axis (slit direction). This effect is the optical equivalent to "beam smearing" in radio observations. To investigate this effect, we measured $\mathrm{RCs}$ for slit widths ranging from $1 \mathrm{kpc}$ to $10 \mathrm{kpc}$, which corresponds for our model galaxy A to $0.2 h^{-1} R_{\mathrm{d}}$ to $2.2 h^{-1} R_{\mathrm{d}}$. In Figs. 6 and 7 we show the RCs for model galaxy A (IC and evolved, respectively) for two very different slit widths (1 kpc and $10 \mathrm{kpc}$ ).

If the slit width is $1 \mathrm{kpc}$, the scatter around the mean velocity in each bin is very small in comparison to the $10 \mathrm{kpc}$ slit. This can be explained in terms of velocity distributions in a bin. As the slit width is increasing, more gas particles can contribute to the measured mean velocity in a bin. In other words, the mean velocity is a superposition of velocity components

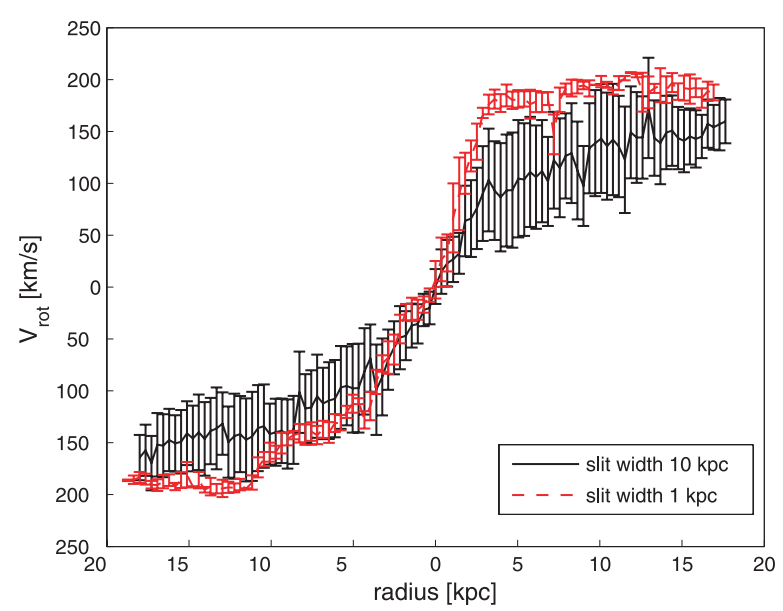

Fig. 7. RCs of model galaxy A after 5 Gyr of evolution for two different slit widths $(1 \mathrm{kpc}$ and $10 \mathrm{kpc})$. The error bars indicated in the figure are the standard deviations of the mean velocity in each $0.1 \mathrm{kpc}$ bin, see Fig. 2 (image b).

from different regions of the galaxy, mainly due to the line of sight velocity distribution. In Fig. 7 the same quantity is shown as in Fig. 6, but after 5 Gyr of evolution of the model galaxy A. Again the same behaviour in the scatter and mean velocity is present, but the overall velocity field shows more structure, which is a consequence of the fully hydrodynamic treatment of the galaxy. Note that ICs (as analytic models) do not include prescriptions for spiral arms, which are present in observed galaxies. Only the $N$-body/SPH simulations can reproduce this feature; therefore the measured ideal $\mathrm{RC}$ for the evolved galaxies shows local fluctuations connected to e.g. spiral arms. This fact is well known from observations, where fluctuations of a few tens of $\mathrm{km} / \mathrm{s}$ are superposed on the smooth rotation curve of the galaxy due to spiral arms (see e.g. Sofue \& Rubin 2001). This agrees well with our model RCs (cf. Figs. 6 and 7).

As a next step we fit Eq. (1) to the RCs extracted from different slit widths, shown in Fig. 8. We used galaxy model A after 5 Gyr of evolution. The galaxy was always "observed" with an inclination $i=80^{\circ}$ and with an ideal spatial resolution of $0.1 \mathrm{kpc}$. As the slit width increases, the fitted curves decrease. Again this can be explained by the averaging process. It is obvious that slits that are too wide $\left(d>R_{\mathrm{d}}\right)$ result in non-flat RCs, which should not be fitted by Eq. (1). Instead an observer would use the URC here. If we adopt our fitting procedures and extract $V_{\max }$ and $V_{\text {opt }}$ for different slit widths, we obtain a dependence of $V_{\max }$ and $V_{\text {opt }}$ on $d$, as shown in Fig. 9. A nearly linear decrease of $V_{\max }$ and $V_{\text {opt }}$ from $d=1 \mathrm{kpc}$ to $d=4.5 \mathrm{kpc}$ can be seen. From this investigation we would recommend applying only a maximum slit width on the order of $R_{\mathrm{d}}$.

\subsection{Rotation curves as a function of inclination}

Galaxies can very rarely be observed edge-on so a correction of the inclination effects on the RC is important. The intrinsic RC $V^{\text {int }}(r)$ of a galaxy is most often corrected by the sine of the inclination angle, i.e. by the simple geometric correction $V^{\text {int }}(r)=V^{\text {obs }}(r) / \sin (i)$ (edge-on galaxies are defined as having $i=90^{\circ}$ ). We investigated the $\mathrm{RC}$ of model galaxy A by rotating 


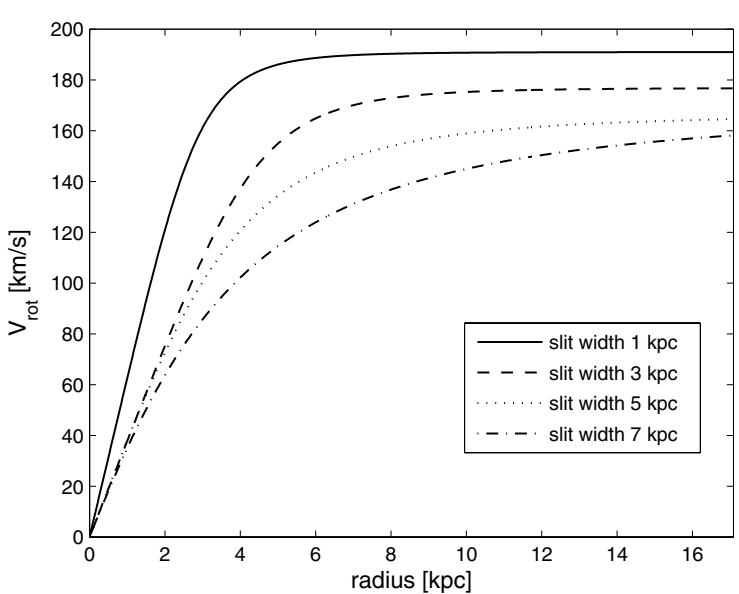

Fig. 8. Best fits to RCs for different slit widths. The underlying galaxy model is A after 5 Gyr of evolution.

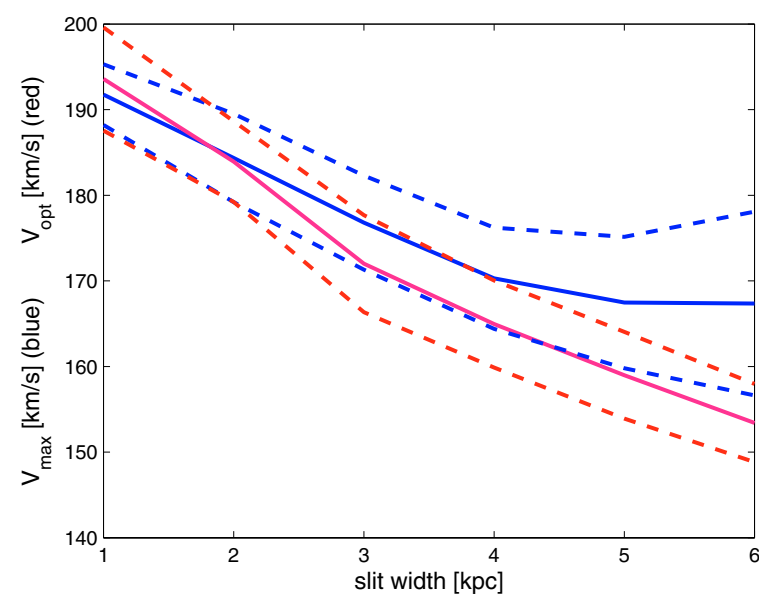

Fig. 9. Fitting parameters $V_{\max }$ (blue) and $V_{\text {opt }}$ (red) as a function of slit width for model galaxy A with ideal spatial resolution. The dashed lines correspond to the bounds of the $95 \%$ confidence level. After an almost linear decrease, the scatter increases for $V_{\max }$.

the galaxy from $i=20^{\circ}$ to $i=80^{\circ}$, which is a typical range of inclination angles accessible in observations. The slit was centred on the gas disc with a slit width fixed to $d=4 \mathrm{kpc}$. After each rotation, we extracted the RC, corrected with the simple expression $V^{\text {int }}(r)=V^{\text {obs }}(r) / \sin (i)$ before, and fit the data with Eqs. (1) and (2). The dependence of $V_{\max }$ and $V_{\text {opt }}$ on the inclination angle $i$ is shown in Fig. 10. In the inclination range $80^{\circ}>i>70^{\circ}, V_{\max }$ and $V_{\mathrm{opt}}$ show a steep increase, while for $i<65^{\circ} V_{\max }$ and $V_{\text {opt }}$ stay roughly constant. The explanation for this behaviour is shown in Fig. 11. If a galaxy is observed nearly edge-on, the rotational velocity is an average of velocities from all radial distances along the line-of-sight. With decreasing inclination, more and more gas particles with lower line-of-sight velocity components move out of the slit, and are therefore not taken into account in the averaging process. Thus, the mean velocity in each bin increases, which again leads to a larger $V_{\max }$ and $V_{\text {opt }}$. Below a certain inclination angle, depending on the slit width, most of the volume of the disc is not covered by the slit. Most gas particles with a low lineof-sight velocity component are located in this volume. In our case, $70 \%$ of the volume is not covered by the slit for $i<65^{\circ}$.

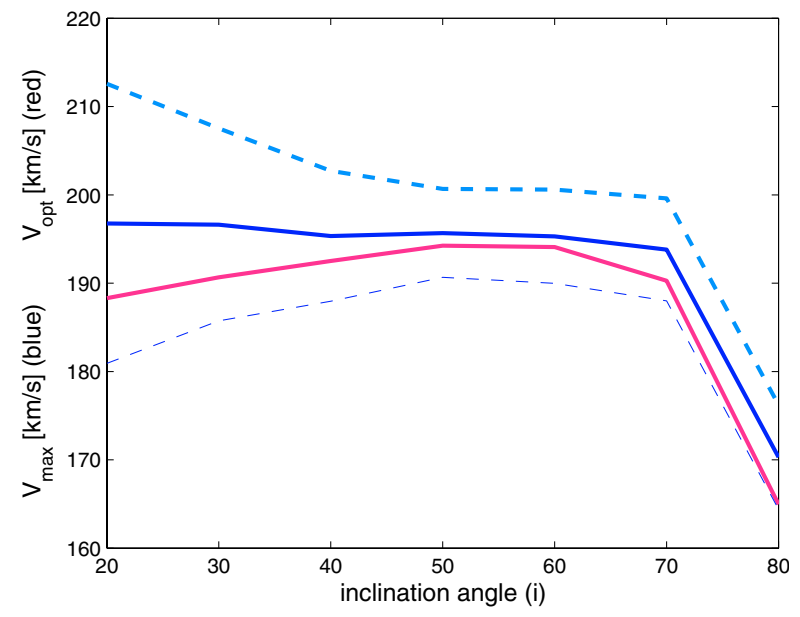

Fig. 10. Fitting parameter $V_{\max }$ (blue) and $V_{\text {opt }}$ (red) as a function of the inclination angle. The dashed lines correspond to the bounds of the $95 \%$ confidence level of $V_{\max }$. The underlying model is galaxy A after $5 \mathrm{Gyr}$ of evolution. A constant slit width of $4 \mathrm{kpc}$ was used.

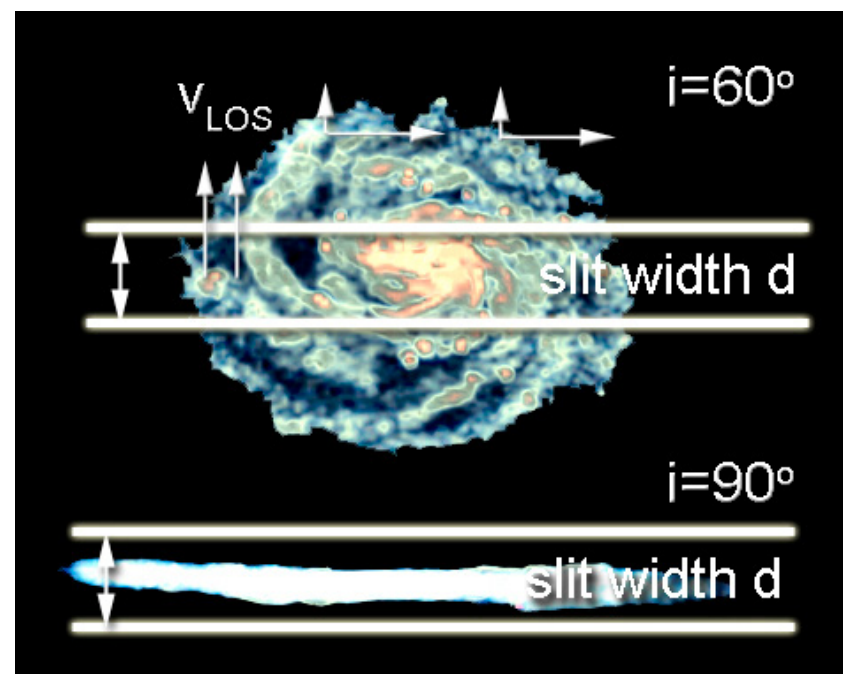

Fig. 11. Sketch of the influence of the inclination on a spiral galaxy for a fixed slit width for model galaxy A.

Note that this behaviour is most significant for large relative slit widths. In observations one tries to overcome the problem occurring at high inclination angles by special techniques, as e.g. the "envelope-tracing" method (e.g. Sofue \& Rubin 2001).

As the inclination angle $i$ decreases the errors for the fit become larger. Note that for $i<35^{\circ}$, the errors are on the order of $10 \%$. In Fig. 12 we show the fitting parameter $V_{\max }$ as a function of the inclination angle $i$ for different slit widths. If the inclination is below $70^{\circ}$ and above $30^{\circ}$, the slit width does not affect $V_{\max }$. The same behaviour was found for $V_{\text {opt }}$. Only in the cases near edge-on and face-on, the slit width plays an important role in covering gas particles. The overall trend is the same as shown in Fig. 9, where larger slit widths lead to lower $V_{\max }$ and larger errors.

Therefore, for large inclinations $\left(i>70^{\circ}\right)$ the slit width varies $V_{\max }$ strongly (see Figs. 11 and 12), while for smaller inclinations the determination of $V_{\max }$ does not strongly depend on the slit width. 


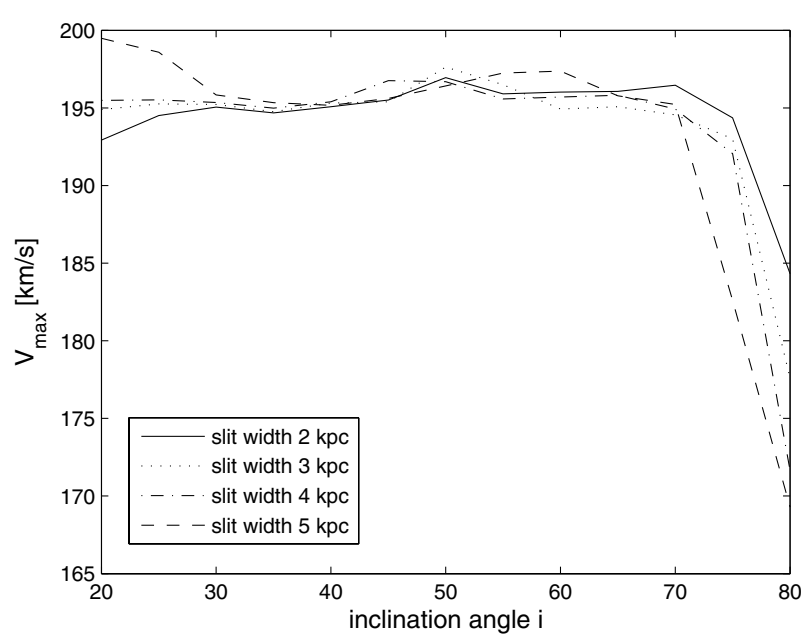

Fig. 12. Fitting parameter $V_{\max }$ as a function of the inclination angle, for slit widths in the range of $2 \leq d \leq 5 \mathrm{kpc}$ for model galaxy A.

\subsection{Rotation curves as a function of misalignment}

As shown in Fig. 1, the slit for measuring the RCs can have a misalignment $\delta$ with respect to the major axis of the projection of the galaxy on the sky. Multi-object spectroscopy, especially, has to deal with misaligned slits, therefore we investigated the effect of $\delta$ on the fitting parameters $V_{\max }$ and $V_{\text {opt }}$. In Fig. 13 we plot the result for different $\delta$ for a fixed slit width of $d=4 \mathrm{kpc}$ for model galaxy A. Note that we applied the standard $\cos ^{-1}(\delta)$ correction. Nevertheless the fitting parameters $V_{\max }$ and $V_{\text {opt }}$ are not independent of $\delta$. The corresponding error (scatter around the mean velocity in a bin), does not show any dependence on the misalignment angle $\delta$, if it covers particles all along the slit. As the $\cos (\delta)$ correction we applied is only fully valid for two-dimensional discs without any thickness, $V_{\max }$ and $V_{\text {opt }}$ show a dependence on $\delta$, the slit misalignment. In the case of multi-object spectroscopy, where the misalignment can be much higher, more advanced corrections have to be applied. We introduced one method in Böhm et al. (2004).

\subsection{Rotation curves as a function of binning}

To simulate different spatial resolution we binned our ideal RC, see Fig. 3. In Fig. 14 we show the dependency of the fitting parameters $V_{\max / o p t}$ on different spatial resolutions, together with standard deviations. Obviously a poor spatial resolution leads to larger errors, but the value $V_{\max }$ shows no dependence. As $V_{\max }$ represents the flat part of the RC (the asymptotic velocity), the binning does not make $V_{\max }$ vary while it does of course for $a$ and $r_{0}$.

\subsection{Estimators for the virial mass}

One major goal of investigating RCs is the possibility of determining the mass of the system including baryonic and nonbaryonic components. The virial mass of a galaxy is $\propto$ to $V_{\mathrm{c}}^{3}$ (e.g. Mo et al. 1998), where $V_{\mathrm{c}}$ is the rotational velocity of the galaxy at the virial radius. Unfortunately, it is not

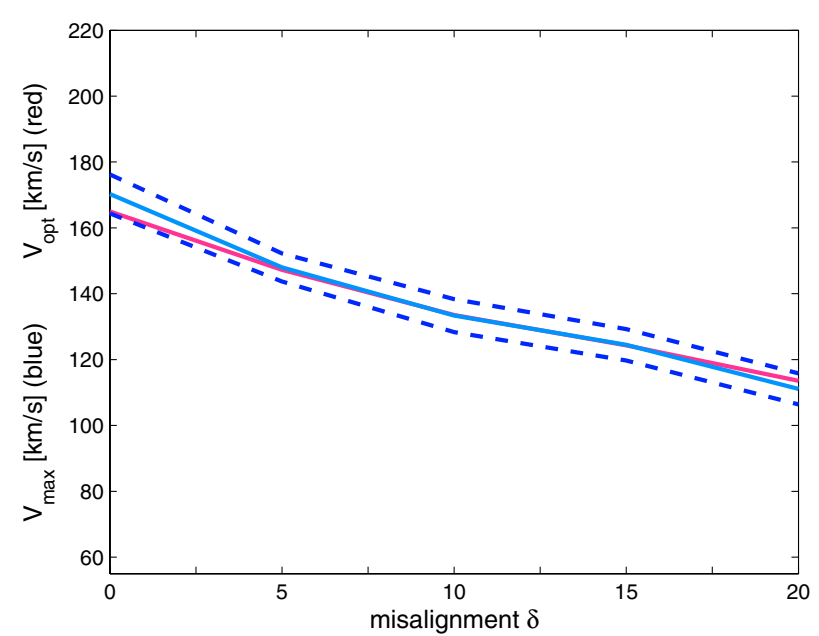

Fig. 13. Fitting parameters $V_{\max }$ (blue) and $V_{\text {opt }}$ (red) as a function of the misalignment angle for a fixed slit width of $d=4 \mathrm{kpc}$ for model galaxy A. The dashed lines correspond to the bounds of the $95 \%$ confidence level for the fitting parameter $V_{\max }$.

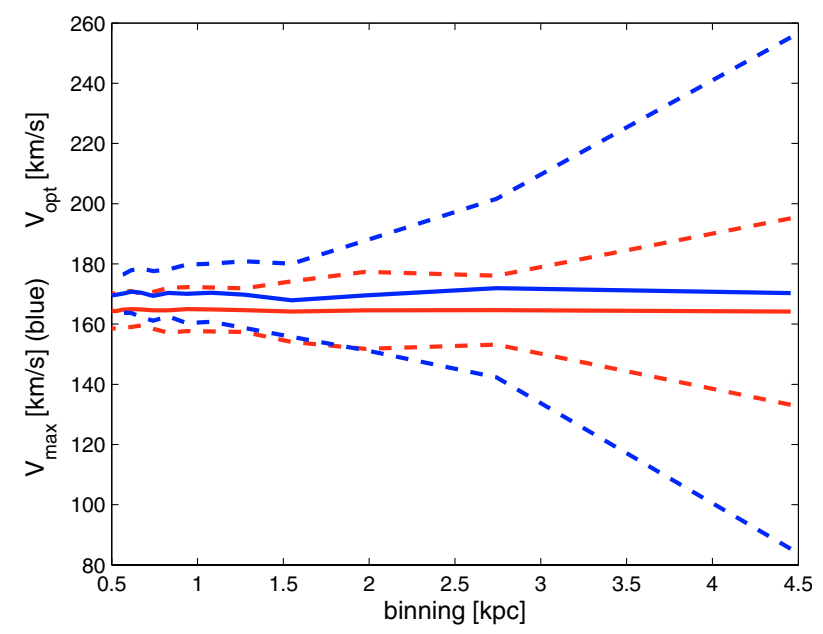

Fig. 14. Fitting parameters $V_{\max }$ and $V_{\mathrm{opt}}$ as a function of the spatial resolution. Obviously the quality of an RC decreases for poorer spatial resolutions.

possible to measure this velocity directly; therefore the virial mass of a galaxy is estimated by some suitable measure of the maximum circular velocity. If $V_{\max }$ converges to $V_{\mathrm{c}}$ at the virial radius, the RC fitting procedure with Eq. (1) is a good estimator of the virial mass. However, as $V_{\max }$ depends on observational constraints and of internal properties of the galaxy, it seems to be no robust estimator of the total virial mass. Van den Bosch (2002) investigated the impact of cooling and feedback on disc galaxies using an analytical model. The author comes to similar conclusions; however, as he does not treat the gas selfconsistently (i.e. fully hydrodynamically), he cannot derive a rotation curve and $V_{\max }$ as we do. Instead in van den Bosch's work, $V_{\max }$ is defined as the maximum rotation velocity inside the radial extend probed by the cold gas (van den Bosch 2002). 
The author finally concludes that Eq. (3) seems to be a good fitting function to obtain the virial mass of a system:

$M_{\mathrm{vir}}=2.54 \times 10^{10} M_{\odot}\left(\frac{r_{\mathrm{d}}}{\mathrm{kpc}}\right)\left(\frac{V_{\max }}{100 \mathrm{~km} \mathrm{~s}^{-1}}\right)^{2}$.

Van den Bosch (2002) did not intend to give an exact estimator of the virial mass by this fitting function. Instead, it seems that he wanted to use $V_{\max }$ to give a rough estimate for the influence of cooling and feedback on the determination of the virial mass. Therefore, it is clear that Eq. (3) is not exact. Nevertheless, as some observers use this function, we also applied it to our simulated galaxies.

As we treated the galaxies fully hydrodynamically, we were able to show the influence of evolution on the RCs. The general decrease of $V_{\max }$ is given in Fig. 4. The main element to decrease the angular momentum is the presence of a galactic wind that ejects matter from the disc into the surrounding halo. In most cases we find that $V_{\max }$ derived from the fitting procedures does not represent $V_{\mathrm{c}}$ and is also different from the maximum rotation velocity present in the full velocity field. Most determinations of $V_{\max }$ (different inclination, slit misalignments, and slit widths) result in an overestimation of $V_{\mathrm{c}}$, if the standard corrections $\sin (i)$ and $\cos (\delta)$ are applied. The virial mass of our model galaxies, obtained by extracting $r_{200}$ as limiting radius, is $M_{200}=1.13 \times 10^{12} M_{\odot}$ for model galaxy A and $M_{200}=1.44 \times 10^{11} M_{\odot}$ for model galaxy B. We used Eq. (2) to determine the virial mass and to find a systematic underestimation of $\approx 50 \%$. As van den Bosch (2002) states correctly, matter can be ejected by galactic winds and therefore reduce $V_{\max }$ by increasing $R_{\mathrm{d}}$ at the same time. The same behaviour is present in our $N$-body/SPH simulations. However, we find that Eq. (3) underestimates the virial mass of our model galaxies. The same underestimation of van den Bosch's (2002) estimation was stated by Conselice et al. (2005). However, as mentioned earlier, Eq. (3) is not thought to be exact. In fact, van den Bosch (2002) mentions that the error for an individual galaxy can still exceed a factor of 2 . Thus it is no surprise that the result is not correct for our model galaxy.

A more detailed mass decomposition by applying the URC fitting would allow a deeper insight into the mass distribution of a galaxy. Nevertheless, the same problems would be inherent from the observational point of view, such as slit width, slit misalignment, or galaxy orientation. The influence of the previously mentioned effects on the mass decomposition by the $\mathrm{RC}$ is being investigated for a future paper. Here we conclude that it is important for observers to investigate the environment of the measured galaxy. As galactic winds can strongly influence the internal kinematics of the gas in the disc knowledge of the star-formation rate puts constraints on the robustness when determining the virial mass from $V_{\max }$. An important issue in this context is whether the galaxy is a member of a group, a galaxy cluster, or the field. It is important to note that especially spiral galaxies in galaxy clusters can often interact with each other. As the interaction (Kapferer et al. 2005) increases the star-formation rate significantly, merger-driven starbursts occur for a short time (up to several 100 million years) and expel huge amounts of interstellar matter into the surrounding halo.
These systems, therefore, might have lower rotational velocities than isolated, low star-forming galaxies, while the general shape of the RC remains similar.

\subsection{The Tully-Fisher relation of spiral galaxies}

Semi-analytical galaxy-formation models usually fix their free parameters such that the models match observed present-day luminosity functions (LF) or TFRs. Early models had problems predicting the correct LF at the same time as the correct zero point of the TFR; therefore, additional physical processes were introduced to fit both important statistical properties of a galaxy population at the same time. However, most semi-analytical models only have rough models for approximating the circular velocity of the halo $V_{\mathrm{c}}$, to derive a TFR for the underlying galaxy population. This, in turn, can differ significantly from the maximum rotational velocity $V_{\max }$, derived from observed RCs, as discussed in the previous sections. Thus, differences of the zero point of observed and modelled TFRs could, at least to some extent, originate from these discrepancies of $V_{\max }$ and $V_{\mathrm{c}}$.

We do not claim here that the differences between $V_{\max }$ and $V_{\mathrm{c}}$ solve the problem of the zero point discrepancies of observed and simulated TFRs. We merely want to point out that these two quantities are in general not equal and therefore it is problematic to use them equivalently. A major contribution to the difference between $V_{\max }$ and $V_{\mathrm{c}}$ are galactic winds, as they are able to decrease the angular momentum of a disc by expelling a significant amount of matter into the surrounding halo. As we are now able to show that $V_{\max }$, for given modelled galaxies and derived as in observations, indeed mostly overestimates $V_{\mathrm{c}}$, we emphasise the importance of cooling and feedback processes. We plan to investigate the TFR of a semianalytical model in a forthcoming work, taking this discrepancy into account. The effect of the overestimation of $V_{\max }$ as $V_{\mathrm{c}}$ is sketched in Fig. 15. If $V_{\max }$ was equal to $V_{\mathrm{c}}$, we would expect that observed and simulated TFRs coincide in the right line. If $V_{\max }$ is higher than $V_{\mathrm{c}}$, the TFR of the simulations would be located at the position of the left indicated line.

It is important to stress that the direct comparison of observation $\left(V_{\max }\right)$ and simulation $\left(V_{\mathrm{c}}\right)$ does invoke uncertainties originating from internal kinematics of the disc, like galactic winds. Similar concerns were emphasised by van den Bosch (2000). Tully Fisher relations can also be constructed by using $V_{\text {opt }}$. Again the same observational constraints would introduce uncertainties, but the increase in the disc scale length due to galactic winds is compensated for by measuring $V_{\text {opt }}$ at a given optical radius. Different disc scale lengths result in different optical radii; therefore, the evolution of the gaseous disc is taken into account.

\section{Summary and conclusion}

In this work we used fully hydrodynamically modelled galaxies, which include star formation, stellar feedback, and galactic winds to study the internal kinematics of the gas in a spiral galaxy. We extracted an RC from the line-of-sight velocities of the gas particles. Then we used a three-parameter fitting 


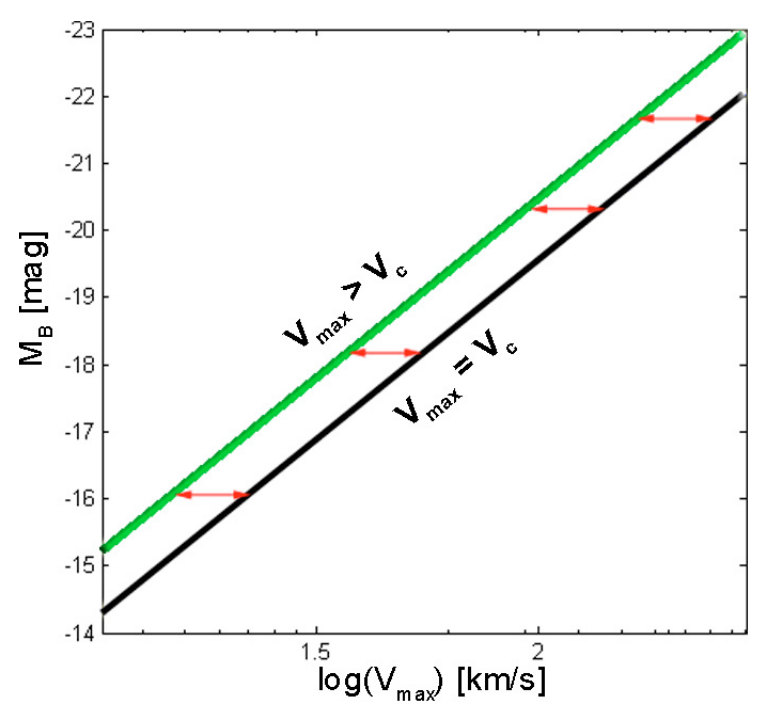

Fig. 15. Sketch of a shift in the Tully-Fisher relation due to discrepancies between $V_{\mathrm{c}}$ and $V_{\max }$. Only if the assumption $V_{\max }=V_{\mathrm{c}}$ holds, Tully-Fisher relations from semi-analytical models and from observations are comparable. If $V_{\max }>V_{\mathrm{c}}$ (result of our investigation) the line of the semi-analytical model would be shifted to the left with respect to the observed line.

formula to describe the rotation curve (Courteau 1997) and the universal rotation curve (URC, Persic et al. 1996). We find

- For evolved model galaxies, the extracted and fitted RCs show a tendency towards lower rotational velocities, compared to the initial conditions. This can be explained by galactic winds, which expel a certain amount of matter into the surrounding halo and therefore decrease the total angular momentum.

- We show that the variation of the slit width does influence the quality of the RC and the values of $V_{\max }$, as well as of $V_{\text {opt }}$. If the slit width is small, $V_{\max }$ and $V_{\text {opt }}$ are systematically higher and show less scatter. If the slit width is large, the mean velocity is a superposition of particles along the line of sight, which introduces low velocity components and therefore larger scatter.

- The dependence of $V_{\max }$ and $V_{\text {opt }}$ on the inclination angle is nearly constant over the range of $20^{\circ}<i<70^{\circ}$. In the range above $i=70^{\circ}$, both show a strong dependence on $i$, which can be explained by rotating velocity components from the foreground and background of the disc into the slit. It turned out that the sine correction leads to similar results in the range of $20^{\circ}<i<70^{\circ}$. In this range we did not encounter strong dependencies on the slit width.

- The $\cos (\delta)$ correction for the slit misalignment is only fully valid for two-dimensional discs, without any thickness. Thus, $V_{\max }$ and $V_{\text {opt }}$ show an almost linear dependence on $\delta$. In the case of multi-object spectroscopy, where misalignments are inherent, more advanced corrections have to be applied, such as those introduced by Böhm et al. (2004).

- The spatial resolution does not influence $V_{\max }$ and $V_{\mathrm{opt}}$ strongly, but influences the quality of the RC.

- We tested the capability of $V_{\max }$ as an estimator for the virial mass of the system and found a strong overestimation of the virial mass by applying the virial theorem. By testing a more sophisticated relation, including results of semianalytic models introduced by van den Bosch (2002), we found an underestimation on the order of $50 \%$ of the virial mass. The explanation for the disagreement with van den Bosch lies in the fully $N$-body/hydrodynamic treatment in our simulations. Another point is the discrepancy of deriving $V_{\max }$, in our case from RCs from our model galaxies and in his case with the semi-analytical approach.

- As Tully-Fisher relations are a common tool for testing models of galaxy evolution, any systematic differences between observations and theory play important roles. We have shown that $V_{\text {max }}$ and $V_{\text {opt }}$ usually differ from $V_{\mathrm{c}}$, which introduces a shift into the Tully-Fisher relation.

Investigation of the influence of minor/major mergers and galaxy flybys is an important issue within this topic, which will be discussed in a forthcoming paper.

Acknowledgements. The authors would like to thank Volker Springel for providing GADGET2 and his initial condition generator. The authors are grateful to the anonymous referee for his/her criticism that helped to improve the paper. The authors acknowledge the Austrian Science Foundation (FWF) through Grant Number P15868, the UniInfrastrukturprogramm 2004 des bm:bwk Forschungsprojekt Konsortium Hochleistungsrechnen, the bm:bwk Austrian Grid (Grid Computing) Initiative, the Austrian Council for Research and Technology Development, and the German Science Foundation (DFG) through Grant Number Zi 663/6-1. In addition, the authors acknowledge the Deutsches Zentrum für Luft- und Raumfahrt through Grant 50 OR 0301, the ESO-Mobilitätsstipendien des bm:bwk (Austria), and the Tiroler Wissenschaftsfonds.

\section{References}

Babcock, H. W. 1939, Lick Observatory Bulletin, 19, 41

Böhm, A., Ziegler, B. L., Saglia, R. P., et al. 2004, A\&A, 420, 97

Conselice, C. J., Bundy, K., Ellis, R. S., et al. 2005, ApJ, 628, 160

Courteau, S. 1997, AJ, 114, 2402

Dickinson, M., Papovich, C., Ferguson, H. C., \& Budavári, T. 2003, ApJ, 587, 25

Gabasch, A., Bender, R., Seitz, S., et al. 2004, A\&A, 421, 41

Kapferer, W., Knapp, A., Schindler, S., Kimeswenger, S., \& van Kampen, E. 2005, A\&A, 438, 87

Mihos, J. C., \& Hernquist, L. 1994, APJ, 437, 611

Mo, H. J., Mao, S., \& White, S. D. M. 1998, MNRAS, 295, 319

Persic, M., Salucci, P., \& Stel, F. 1996, MNRAS, 281, 27

Persic, M., \& Salucci, P. 1991, ApJ, 368, 60

Sofue, Y., \& Rubin, V. 2001, ARA\&A, 39, 137

Springel, V., \& Hernquist, L. 2002, MNRAS, 333, 649

Springel, V., Di Matteo, T., \& Hernquist, L. 2005, MNRAS, 361, 776

Springel, V. 2005, MNRAS, submitted [arXiv: astro-ph/0505010]

Tully, R. B., \& Fisher, J. R. 1977, A\&A, 54, 661

van den Bosch, F. C. 2000, APJ, 530, 177

van den Bosch, F. C. 2002, MNRAS, 332, 456

Yegorova, I., \& Salucci, P. 2004, Baryons in Dark Matter Halos

Ziegler, B. L., Böhm, A., Fricke, K. J., et al. 2002, ApJ, 564, L69

Ziegler, B. L., Böhm A., Jäger, K., Heidt, J., \& Möllenhoff, C. 2003, ApJ, 598, L87 